\title{
The Temperature Dependence of the NiTi Long-Range Order Parameter from Non-Empirical Approach
}

D. Fuks and S. Dorfman*

Materials Engineering Dep., Ben-Gurion University of the Negev, P.O. Box 653, 84105 Beer Sheva, Israel

* Dep. of Physics, Israel Institute of Technology-Technion, 32000 Haifa, Israel

\begin{abstract}
On the example of the intermetallic phase we illustrate the ability of the nonempirical methods in the description of the ordering process. The suggested straigtforward scheme of evaluating the generalized mixing potential permits us to reproduce the thermodynamics of the ordering with the high accuracy without any preconceived simplifications. The calculated potentials may be used for description of the phase relations.
\end{abstract}

\section{INTRODUCTION}

Martensitic phase transformations are temperature induced structural phase transitions. These transformations are characterized by collective shifts of atoms leading to the reconstruction of the lattice from the high-temperature austenitic to low-temperature martensitic phase. For ordered intermetallic compounds this process may be accompanied by the ordering of atoms. The thermodynamic description of such a transformation is based on the investigation of the temperature behaviour of thermodynamic functions and has to account the changes of the long-range order parameter in these phases.

The study of thermodynamic properties from the first principles is a complicate question and additional approximations to the electronic theory are usually introduced. These approximations for the completely disordered phases are not coinside with those applied to the ordered phases. For example, the Coherent Potential Approximation (CPA) is well defined for absolutely disordered solid solutions but is inapplicable to the calculations of the thermodynamic functions of the ordered state. Contrary to this applicability of traditional non-empirical schemes for the band structure calculations of intermetallides for the partially disordered phase is questionable. The reason is that 
with the growing temperature there appears the temperature dependent probability to find an atom of any sort on the lattice sites. The solution herewith is connected with the expansion on a number of the structure simplexes (so called, Cluster Variation Method - CVM). The question on this way is evident: how much terms could be included into this expansion for the reproducing the long-range metal or ionic interactions. The appropriate answer is following: from 3 up to 30 terms. In each case this answer is based on the detailed study of convergency of expansion series.

A study of phase competition of different phases in a wide concentration region demands the concentration dependent effective potentials of interatomic interactions. A problem also arises in the case when the homogeneity regions of the ordering phases are wide. Another situation that represents such a necessity is a well known fact of existance of the ordering phases with the same symmetry but different temperatures of the order-disorder phase transition (as $C u_{3} A u$ and $C u A u_{3}$ ). Such phases are represented by the same superstructure vector $\vec{k}_{s}$, and the difference in the orderdisorder transition temperature, $T_{c}$, is connected with the concentration dependence of interatomic potentials. So for the description of such situations one has to evaluate the concentration dependence of the mixing potential. The procedure described in Ref.[1] for the estimation of the parameters of the effective mixing potential may be realized for different phases with the stoichiometric composition. Taking into consideration, for example, only phases that are stable from the Lifshitz criterion[2] and writing down the formulaes, analogous to those represented for the $\mathrm{B} 2$ phase in the ground state, it is possible to evaluate the effective mixing potential for the different concentrations, corresponding to the stochiometric compositions.

We choose NiTi (B2-type) phase as an example demonstrating the advantages of our approach. NiTi undergoes a complicated chain of phase transitions. Recently a new phase transition phenomena in NiTi alloy was reported[3]. This material stimulated a lot of theoretical papers devoted to the analysis of phase stability for different NiTi structures. As an example of the first-principle study of phase stability of NiTi we refer to Ref.[4] and references therein, while semi-empirical tight-binding models are discussed and applied to this alloy in Ref.[5].

In the approach that we are suggesting in our paper the description of the temperature dependences of the long-range order parameter and of the free energy of ordering will be done according to the static concentration wave (SCW) theory[6]. The SCW method allows to take into account interatomic interactions at arbitrary distances. It establishes the relation between the statistical theory and the Landau-Lifshitz thermodynamic theory of second order transformations in alloy ordering. This method provides the possibility of prediction of the structure of the ordered phase if the pairwise interatomic interactions are estimated. Thus SCW theory overcomes several principal difficulties of the traditional theories of ordering[7,8]. We will combine the SCW theory with the firstprinciples calculations of the interatomic interaction and evaluate the above mentioned temperature dependences as well as the temperature of order-disorder phase transformation. 


\section{DERIVATION OF THE THERMODYNAMIC PARAMETERS}

In the framework of the SCW theory the internal mixing energy of absolutely ordered phase $U$ may be written in terms of pairwise interactions

$$
U=\frac{1}{2} V(0) c^{2}+\frac{1}{2} \sum_{s} \gamma_{s}^{2} \eta_{s}^{2} V\left(\vec{k}_{s}\right)
$$

Here $c$ is the concentration of the component $A$ in the ordered phase, $\eta_{s}$ - are the long-range order parameters, that are describing the ordering in the superstructure, $\gamma_{s}$ are the structural constants depending on the symmetry of the ordering phase. $V\left(\vec{k}_{s}\right)$ is the Fourier transform of the mixing potential $V(R)=V_{A A}(R)+V_{B B}(R)-2 V_{A B}(R)$ that is calculated in the superstructure reciprocal lattice vectors $\vec{k}_{s}$

$$
V\left(\vec{k}_{s}\right)=\sum_{\vec{R}} V(\vec{R}) e^{i \vec{k}_{s} \vec{R}},
$$

$V(0)$ is just the same as (2) but for $\vec{k}_{s}=0$. Vectors $\vec{k}_{s}$ belong to the stars of vectors describing the superstructure. Summation in Eq.(2) is produced over the stars of vectors satisfying the Lifshitz criterion[2]. In the case of CsCl-type (B2) superstructure, for example the vector $\vec{k}_{s}$ that is responsible for the formation of this phase from the disordered $b c c$ solid solution is $\vec{k}_{s}=2 \pi / a(111)$. As shown in Ref.[6] $\gamma_{s}=\frac{1}{2}$ for this structure. The vector $\vec{k}_{s}$ defines the site occupation probabilities for the ordered phase that is stable regarding to the formation of the antiphase domains in an alloy. The site occupation probability, $n(R)$, for $\mathrm{B} 2$ superstructure is

$$
n(R)=c+\frac{1}{2} \eta e^{2 \pi i(x+y+z)}
$$

where $x, y$, and $z$ are the coordinates of the bcc lattice sites. At $c=\frac{1}{2}$ and $\eta=1$ this equation describes the completely ordered phase. With Eq.(3) for this $\vec{k}_{s}$ it is easy to obtain

$$
V\left(\vec{k}_{s}\right)=-8 V\left(R_{1}\right)+6 V\left(R_{2}\right)+12 V\left(R_{3}\right)-24 V\left(R_{4}\right)+8 V\left(R_{5}\right)
$$

and

$$
V(0)=8 V\left(R_{1}\right)+6 V\left(R_{2}\right)+12 V\left(R_{3}\right)+24 V\left(R_{4}\right)+8 V\left(R_{5}\right)
$$

Here we restricted ourselves by only five coordination shells of the Ising lattice. Now we can get the expression for the stoichiometric composition of B2 phase energy in the form

$$
U=\frac{3}{2} V\left(R_{2}\right)+3 V\left(R_{3}\right)+2 V\left(R_{5}\right)
$$

It is obvious that this mixing energy in the ground state depends only on the interaction potential between atoms in such a binary phase. If such potential is known it is possible to calculate the temperature dependence of the long range order parameter using the equation of the Bragg-Williams (BW) type

$$
\ln \left[\frac{(1-c-1 / 2 \eta)(c-1 / 2 \eta)}{(1-c+1 / 2 \eta)(c+1 / 2 \eta)}\right]=\frac{V\left(\vec{k}_{s}\right)}{k T} \eta,
$$

and the temperature of the order-disorder phase transition 


$$
T_{c}=-\frac{c(1-c) V\left(\vec{k}_{s}\right)}{k} .
$$

Here $k$ is the Boltzman constant. Substituting $\eta(T)$ dependence into Eq. (1) one can immediately obtain the temperature dependence of the internal energy.

\section{DISCUSSION}

To carry out the potential V(R) that is the keystone of such a calculation we use the Full Potential Linearized Muffin-Tin Orbitals method (FP LMTO). We calculate the total energies of B2 phase NiTi as well as the total energies of the pure $\mathrm{Ni}$ and pure Ti metals in the bcc lattice for different volumes, $\Omega$, per atom and get $U$ vs $\Omega$ dependence. The equilibrium lattice parameter for studied NiTi phase is $2.90 \AA$ and it is in agreement with experiment[10]. The internal mixing energy corresponding to the equilibrium volume, $0.061 R y / a t$, agrees well with the measurements of the enthalphy of formation of $\mathrm{NiTi}$ (B2), $0.051 R y / a t[11]$. The correct sign of the calculated energy of formation is the additional confirmation of the validity of the calculation procedure. Contrary to our results, the authors of the smart modelling of some of $\mathrm{Ni}$-Ti phases[4] obtained the positive formation energy for the B2 phase. The negative formation energy indicates the stability of this intermetallic compound with respect to the phase separated alloy.

These results encourage us to make predictions of the temperature dependences of the long-range order parameter and of the free energy of this phase. With the assumed Morse-type potential form of the effective pair interaction (see, for example [12])

$$
V(R)=A-2 B e^{-\lambda\left(R-r_{0}\right)}+B e^{-2 \lambda\left(R-r_{0}\right)},
$$

we obtained the potential of mixing and from Eq.(6) we estimated from the fitting $U(\Omega)$ procedure the parameters $A, B, r_{0}$ and $\lambda$ of this potential. These values are $A=0.0042 R y, B=0.0421 R y$, $r_{0}=4.7506 \mathrm{a}$.u. and $\lambda=0.7069 \mathrm{a} . \mathrm{u}^{-1}$. Taking into account Eq.(4) the value was obtained $V\left(k_{s}\right)=$ $-0.0822 R y$, that gives the temperature of phase transformation $T_{c} \simeq 3410 \mathrm{~K}$. This temperature is high in comparison with the melting temperature of $\mathrm{B} 2$ phase in our case $T_{m}=1583 \mathrm{~K}$, thus showing that the melting of the phase starts ealier than the order-disorder transformation. Nevertheless the disordering effects may be calculated and the temperature dependence of the long-range order parameter is presented on Fig.1. Direct evaluation of $V\left(\vec{k}_{s}\right)$ values from the $U(\Omega)$ dependence is not obvious because $V(0)$ and $V\left(\vec{k}_{s}\right)$ are entering the Eq.(2) in a symmetric form for the absolutely ordered B2-phase in stoichiometric composition. Eq.(2) for this case has the form

$$
U=\frac{1}{8} V(0)+\frac{1}{8} V\left(\vec{k}_{s}\right)
$$

that leads to the arbitrary evaluation of $V\left(\vec{k}_{s}\right)$ and the estimation of the transition temperature 
becomes nonunique.

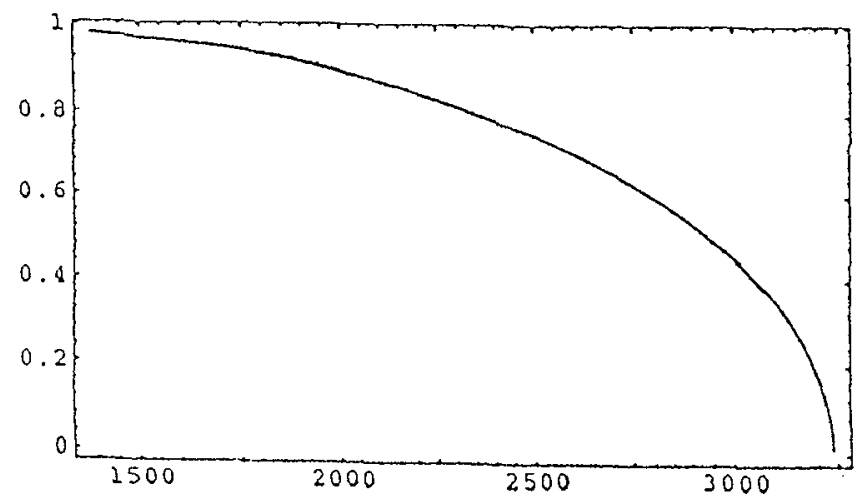

Fig.1: The temperature dependences of the long-range order parameter, $\eta$, for $N i T i$

\section{SUMMARY AND CONCLUSION}

Heretofore we have studied the applicability of the effective potential approach based on the non-empirical calculations to the problem of evaluating of the energetic parameters of the ordering process. The scheme for calculation of the volume dependence of the internal mixing energy was suggested and for simplicity was illustrated on the example of B2-phase (NiTi). The parameters of the effective potential were estimated by fitting to the mixing energy. The latter allows to perform calculations of the temperature dependence of the long-range order parameter in the framework of static concentration waves theory of Khachaturyan[6]. In contradiction to the traditional approaches for the description of the ordering process we do not evaluate the partial pair potentials in our schene. Using this formalism we have demonstrated the principal possibility of the prediction of the temperature of the order-disorder phase transition. 'The quality of this prediction in the case of NiTi can be proved from the $X$-ray measurements of the diffuse scattering.

The stability of the NiTi B2-phase and the existence of the very narrow homogeneity region up) to the melting temperature is a well-known experimental fact and here we want only to illustrate the ability of the calculation of the disordering effects even in this case.

\section{ACNOWLEDGMENTS}

This research was supported in part; by the Israel Ministry of Science and Technology under Grants No.3616 and 4868 and in part by the special program of the Israel Ministry of Absorbtion. We are acknowledging an cnourmous support and discussions during the FP-LMTO calculations with Dr. S.Yu.Savrasov. One of the authors (D.F.) is thankfull to Rashi Foundation for his Guastalla Fellowstip. 


\section{REFERENCES}

[1] D.Fuks and S.Dorfman, Solid State Commun. 90, 773 (1994).

[2] E.M.Lifshitz, Fiz. Zh., 7, 61 (1942); Fiz. Zh., 7, 251 (1942)

[3] Z.C.Lin, P.C.W.Fung, J.X.Zhang, and K.F.Liang, Solid State Commun, 75, 389 (1990).

[4] M.Sluiter, P.E.A.Turchi, F.J.Pinski, and G.M.Stocks, Mater. Science and Eng., A152, 1 (1992).

[5] P.Mikušik and Š.Pick, Solid State Commun., 86, 467 (1993).

[6] A.G.Khachaturyan, Theory of Structural Transformations in Solids, (John Wiley, New York, 1983).

[7] W.L.Bragg and E.J.Williams, Proc. Roy. Soc. A145, 699 (1934); A152, 231 (1935).

J.G.Kirkwood, J. Chem. Phys. 6, 70 (1938).

[8] R.Kikuchi, Phys. Rev. 81, 988 (1951); J. Chem. Phys. 60, 1071 (1974).

[9] H.Okamoto and T.B.Massalski, Bull. Alloy Phase Diagrams, 6(3), 229 (1985).

[10] Landolt-Bornstein, Numerical Data and Functional Relationships in Science and Technology, editors K.-H.Hellwege and A.M.Hellwege, v.6 (Springer, Berlin, 1971).

[11] C.J.Smithells, Metals. Reference Book. v.2 (Butterworths, London, 1962).

[12] J.M.Sanchez, J.P.Stark, and V.L.Moruzzi, Phys. Rev. B44, 5411 (1991). 